\title{
Entre adverbes et pronoms, une question de circonstance
}

\author{
Piron, Sophie \\ Département de linguistique, Université du Québec à Montréal \\ piron.sophie@uqam.ca
}

\section{Introduction}

L'étude que nous proposons suit l'évolution du classement attribué à une série de mots (qui, que, quoi, où, quand, comment, combien, pourquoi, en, y) qui relèvent de deux catégories, celle des adverbes et celle des pronoms. Certains de ces mots ont pu osciller entre les deux classements (notamment en, $y$, où et combien). L'étude présentée ici se limite à une période de quelques décennies du XIX ${ }^{\mathrm{e}}$ siècle, des années 1830 aux années 1880. L'objectif poursuivi est de saisir la constitution d'une partie de l'appareil théorique de la seconde grammaire scolaire française. Cette période de la grammaire se met en place au cours des années 1845-1860 (Chervel, 1977). La date de 1844 marque le tournant symbolique entre la première et la seconde phase de la grammaire scolaire (Chervel, 1979). C'est le moment où le complément circonstanciel acquiert pour la première fois un statut grammatical.

La liste de mots dont nous suivrons l'évolution catégorielle et le poids qui leur est accordé dans les grammaires constitue, nous pensons, un indicateur important dans l'évolution de la grammaire française et par conséquent un critère de périodisation de celle-ci. Cet article s'inscrit ainsi dans une série d'études qui tentent de préciser la chronologie du passage de la première grammaire scolaire à la seconde (Piron 2012a, 2012b, à paraître).

Au XVIII ${ }^{\mathrm{e}}$ siècle, Restaut (1732: 87-88) avait déjà associé les questions quand? où? combien? et comment? à la reconnaissance des adverbes de temps, de lieu, de quantité, de qualité / manière, mais ne classait pas encore explicitement ces mots comme adverbes. Il ne proposait par ailleurs pas de question en pourquoi? , la classe des adverbes de cause ou de motif étant absente. Ces adverbes n'étaient pas liés à une fonction spécifique. Plus tard, Beauzée (1767, T. 2 : 58) aura recours aux mots interrogatifs latins de la rhétorique quis, quid, ubi, quibus auxiliis, cur, quomodo, quando, sans traduction en français ni insistance sur l'interrogation. Ces mots sont associés chez Beauzée non pas à l'établissement de la classe de l'adverbe (comme c'était le cas chez Restaut), mais à un classement des compléments lorsque ceux-ci sont envisagés sous l'angle de leur signification (et non pas sous l'angle de l'analyse logique ou grammaticale). La grammaire scolaire du $\mathrm{XIX}^{\mathrm{e}}$ siècle reliera ces mots à des fonctions grammaticales, d'une part les pronoms interrogatifs au sujet, aux compléments direct et indirect, d'autre part les adverbes interrogatifs au complément circonstanciel.

La répartition des mots qui, que, quoi, où, quand, comment, etc. entre adverbes et pronoms au cours des années 1830-1880 permet d'appréhender les changements à l'intérieur de la théorie grammaticale, à une époque où se cristallise le complément circonstanciel. Les circonstances de lieu, de temps, de manière, de quantité qui définissent ce type de complément se trouvent de plus en plus associées au cours des années 1840-1860 à des mots interrogatifs (Piron, à paraître) : les mots où, quand, comment, combien, pourquoi, ainsi que qui, que, quoi, quel, précédés d'une préposition, sont en train d'acquérir un statut capital dans la reconnaissance et la distinction des compléments circonstanciels et indirects. Cette distinction fonctionnelle (compléments indirects - compléments circonstanciels) est fortement tributaire d'une distinction catégorielle (substantif - adverbe) et constitue une pierre angulaire dans le classement des verbes qui aboutira au début du XXe siècle en une scission des intransitifs (ou neutres) en transitifs indirects d'une part et intransitifs d'autre part (Piron 2012c). Par ailleurs, il est intéressant de suivre l'évolution des critères de définition des pronoms et des adverbes, deux catégories de mots qui posent de nombreuses questions quant à leur comportement syntaxique et sémantique : en particulier, les adverbes forment une « classe poubelle » (Chervel $1977: 251)$, une « catégorie fourre-tout » (Guimier 1996: 1). À ce sujet, Benveniste (1966), Ruwet (1968), M. Gross (1986), Creissels (1995), Choi-Jonin (2001) et G. 
Gross (2012), entre autres, soulignent les problèmes de classement de certains adverbes et leur comportement nominal ou pronominal. Beauzée $(1767$, T. $1: 558)$ avait d'ailleurs déjà analysé hier, avant-hier, aujourd'hui, demain, après-demain comme des noms (mais il proposait en même temps que soient des noms des mots comme toujours, jamais).

La présente étude porte sur un corpus d'ouvrages essentiellement scolaires, d'envergures variées (grammaires élémentaires, abrégés, éléments de grammaire, grammaires des commençants, grammaires complètes, traités complets), mais également quelques grammaires savantes. Ces ouvrages ont pour la plupart été publiés en France. Le corpus compte 78 publications et couvre une période d'environ 50 ans (1835-1883).

Le corpus mis sur pied permettra de vérifier si, en parallèle avec l'évolution des compléments circonstanciel et indirect, les grammaires accordent une place de plus en plus importante aux mots interrogatifs (pronoms et adverbes) en tant que parties du discours et aux notions circonstancielles telles que le lieu, le temps, etc.

\title{
2 Les définitions
}

\subsection{Définition de l'adverbe}

Les définitions que les grammairiens du corpus fournissent au sujet de l'adverbe reposent majoritairement sur la notion de modification. En effet, 59 publications y font référence. Parmi celles-ci, la plupart signalent que l'adverbe a la capacité de modifier plusieurs espèces mots, soit le verbe, l'adjectif et l'adverbe lui-même'.

L'adverbe est un mot invariable qui modifie ordinairement un verbe et quelquefois un adjectif ou un adverbe. (Dessiaux, $1838: 61$ )

L'adverbe est un mot invariable qu'on ajoute à un verbe, à un adjectif ou à un autre adverbe pour le modifier. (Jourdan et Castegnier, $1863: 212$ )

Tout en usant de la notion de modification, d'autres publications précisent que l'adverbe modifie le sens ou la signification de certaines espèces de mots $^{2}$. Elles le placent ainsi dans un rôle sémantique qui lui est spécifique et qui diffère de celui d'un complément. Mais surtout, ces grammaires inscrivent pleinement la définition de l'adverbe dans le domaine du sens.

\begin{abstract}
L'adverbe est un mot invariable employé comme complément circonstanciel pour modifier le sens d'un adjectif, d'un verbe-adjectif, et quelquefois d'un autre adverbe, c'est-à-dire pour donner un sens particulier à l'adjectif, au verbe-adjectif ou à l'adverbe auquel il est joint. (Sardou $1841: 67-68$ )
\end{abstract}

L'adverbe est un mot invariable qui se joint à un verbe, à un adjectif et même à un autre adverbe, pour en modifier la signification. (Belèze, $1862: 124-125)$

Les définitions faisant appel à la modification sont parfois accompagnées de notions vraisemblablement mises sur le même plan, celles de la détermination (Olivier, 1857; Sommer, 1861), de l'ajout (Girard, 1862), de la restriction (Landais, 1856). L'analyse sémantique est toujours privilégiée. Cependant, les associations présentées atténuent la différence entre le rôle de la modification et celui de la complémentation, une ambiguïté qui prend place dans «l'élaboration du concept de complément» (Bouard \& Fournier, $2013: 35$ ), encore au XIX ${ }^{\mathrm{e}}$ siècle.

L'adverbe est un mot que l'on ajoute au verbe ou à l'adjectif pour en déterminer ou en modifier la signification. [...] L'adverbe modifie quelquefois la signification d'un autre adverbe [...]. (Sommer, $1861: 97)$

L'adverbe est un mot invariable qui se met ordinairement près du verbe, pour ajouter à sa signification, pour la modifier. (Girard, $1862: 46$ ) 
Ils [les adverbes] modifient, restreignent ou déterminent le sens du mot auprès duquel ils sont placés. (Landais, $1856: 369 \mathrm{a}$ )

Le deuxième type de définition de l'adverbe est présent dans une dizaine ouvrages du corpus et opte non plus pour la modification, mais pour la précision sémantique qu'un adverbe apporte au mot qu'il accompagne : il en fixe la signification, il la détermine, il la précise.

L'adverbe est un mot invariable qui se place avant ou après les verbes et les adjectifs,
pour donner à nos pensées plus de précision, et de clarté. (D'Ablancourt, 1838:52)

Comme l'influence de l'Adverbe se fait sentir tantôt sur l'Adjectif, tantôt sur l'attribut du Verbe, tantôt sur le Verbe lui-même, comme aussi, d'un autre côté, ce qu'il exprime est toujours invariablement la même pensée, nous pouvons dire que l'Adverbe est un mot qui fixe la signification du Verbe, de son attribut ou de l'Adjectif, ou d'un Adverbe. [...] il fixe l'étendue de la signification [...]. (Ganeval, $1862: 224)$

L'adverbe est un mot qui se joint ordinairement au verbe ou à l'adjectif pour en déterminer la signification [...]. (Jullien, $1852: 125$ )

Si l'on s'attarde quelque peu à la chronologie, on constate que la modification de la signification d'un autre mot ainsi que sa détermination sont des approches de l'adverbe qui semblent s'essouffler à partir des années 1860 tandis que la définition générale selon laquelle un adverbe modifie un autre mot perdure tout au long de la période couverte par le corpus.

Le corpus présente finalement une troisième approche dans la définition de l'adverbe, approche selon laquelle cette espèce de mot ajoute de la signification. Il s'agit d'une perspective très peu représentée (Gallien, 1868 ; Jonette ,1869 ; Chassang, 1881) et d'apparition tardive au sein du corpus.

L'adverbe est un mot [...] qui se place avant ou après le verbe, avant l'adjectif ou un autre adverbe, et quelquefois avant un substantif, pour y ajouter une idée de manière, de quantité, de temps, de lieu, etc. (Chassang, $1881: 166)$

Environ un tiers des définitions de l'adverbe font également référence aux notions de circonstances, soit en utilisant ce terme, soit en dressant une liste de notions au rang desquelles la manière est fréquemment citée, soit encore en combinant les deux présentations. L'adverbe est alors décrit le plus souvent comme un mot permettant d'exprimer ou de marquer une circonstance, mais on trouve également qu'il indique les circonstances, voire en ajoute.

Il [l'adverbe] exprime diverses circonstances de temps, de lieu, d'ordre, de manière, de quantité, d'affirmation, de négation, d'interrogation et de comparaison. (Ministère de la Guerre, $1866: 46$ )

Les adverbes servent en général à marquer les diverses circonstances de lieu, de temps, de manières, etc. qui modifient les adjectifs et les verbes [...]. (Beudant, 1840 : 75)

On relèvera l'usage vraisemblablement néologique du verbe circonstancier par une grammaire anonyme datant de 1866. Cela montre à quel point la notion sémantique de circonstance a pris de l'ampleur dans la définition de l'adverbe.

L'adverbe est un mot invariable qui circonstancie le verbe, l'adjectif ou l'adverbe luimême. (DMD, 1866 : 93)

\subsection{Définition du pronom}

La définition du pronom au sein du corpus établi repose largement sur la notion sémantico-syntaxique de remplacement : le pronom tient la place, se met à la place d'un nom; est employé pour un nom; remplace un nom, le représente. Ces notions traversent tout le corpus. Elles confinent assez souvent à la stylistique, un pronom permettant d'éviter la répétition. 


\begin{abstract}
On appelle pronom, tout mot mis à la place d'un nom pour en rappeler l'idée, et éviter une répétition qui serait désagréable à l'oreille. (D’Assier, $1864: 24$ )

Le pronom est un mot qui remplace le substantif et dont il sert à éviter la répétition. (Bentz, $1838: 25$ )

Le pronom [...] est un mot qui représente le nom et en tient lieu. (Bonneau \& Lucan, $1839: 30)$
\end{abstract}

Quelques grammaires restreignent le pronom à la notion sémantique de personne (Boniface 1948, Larousse 1852, Descamps 1852) ou aux notions de personnes et de choses (Guerrier de Haupt, 1842 ; Colart, 1846 ; Roche, 1860 ; Bescherelle, 1856).

Les personnes et les choses représentées s'appellent pronoms, c'est-à-dire pour noms, se mettant à la place des noms qu'ils représentent. (Colart, $1846: 13$ )

Le pronom est donc un mot mis pour un nom de personne ou de chose, qu'on ne veut pas exprimer ou répéter. (Roche, $1860: 22$ )

Enfin, le pronom est défini sous l'angle de la pragmatique (il indique le rôle tenu dans le discours) par une quinzaine d'auteurs, mais cela n'exclut pas la référence au remplacement qu'il opère.

Le pronom est un mot qu'on emploie à la place du nom pour indiquer le rôle que remplit dans le discours la personne qu'il désigne [...]. (Dunglas, $1855: 12-13$ )

on ne peut pas le [le pronom] considérer comme l'équivalent du Nom, il en rappelle le rôle seulement. [...] C'est que le Pronom est un masque, que tout acteur prend pour son rôle dans le langage. [...] Ainsi, le même individu change de Pronom en changeant de rôle. (Ganeval, $1862: 165-170)$

En somme, la restriction notionnelle est un critère de définition nettement moins utilisé pour le pronom (personnes, choses) que pour l'adverbe (circonstances). Le cœur des définitions réside cependant ailleurs : dans la notion de modification (plan sémantique, mais aussi vraisemblablement syntaxique) pour l'adverbe, dans la notion syntaxico-sémantique de remplacement pour le pronom.

\title{
3 Personnes, choses et lieu
}

Comme nous venons de le voir, le pronom et l'adverbe renferment, chez certains auteurs, une définition notionnelle qui les oppose : d'une part, les notions de personne et de chose; d'autre part, celles de circonstances. Si cette différence notionnelle ne constitue pas le cœur de la définition, elle revêt pourtant une importance insoupçonnée puisqu'elle permet de trancher dans certains cas le problème de l'appartenance catégorielle. En effet, elle fonde une distinction catégorielle pour les mots en et $y$ chez un peu moins de la moitié des auteurs ${ }^{3}$. Certains présentent ainsi en et $y$ à la fois dans le chapitre consacré au pronom (généralement au sein de la liste des pronoms personnels de la $3^{\mathrm{e}}$ personne) et dans celui portant sur l'adverbe (dans la liste des adverbes fréquents ou dans celle dédiée aux adverbes de lieu s'il y a un classement sémantique). Au-delà du classement différencié, d'autres grammairiens attirent l'attention sur ce qui fonde la différence. On trouve ainsi, dans le chapitre du pronom ou dans celui de l'adverbe, des sections « Ne confondez pas » consacrées aux mots pouvant appartenir à plus d'une catégorie (ou, où; la, là; etc.) ou des précisions sur le double classement.

En, $y$, sont aussi pronoms personnels quand ils représentent des personnes ou des choses. [...] Mais en, y, sont considérés comme adverbes, toutes les fois qu'ils indiquent le lieu. (D’Ablancourt, $1838: 16$ )

$E n, y$, sont à la fois adverbes et pronoms. En, $y$, adverbes de lieu, signifient de là, là. Ex. : Connaissez-vous Rouen ? J'en arrive et j'y retourne. En, $y$, pronoms, signifient de lui, d'elle, à lui, à elle, etc. Ex. : Pensez-vous à mon affaire ? J'y pense. (Larive \& Fleury, 1883 : 166-167)

La distinction entre adverbes locatifs et pronoms personnels représentant des personnes ou des choses est présente dès le début du corpus et le traverse entièrement sans devenir pour autant un point de théorie 
incontournable. Au-delà des cas du en et du $y$, le classement du où oscille lui aussi, mais entre pronom relatif et adverbe de lieu (voire adverbe interrogatif).

Nous pensons avec de bons grammairiens que les mots où, $y$, sont toujours pronoms. Mais nous croyons devoir nous conformer ici à l'opinion générale en les classant parmi les adverbes lorsqu'ils expriment le lieu. (Capelle-Grimbert 1847 : p. 77)

La liste de pronoms relatifs ou conjonctifs que proposent les grammairiens a l'intérêt de montrer l'importance du concept de lieu dans la répartition des parties du discours. En effet, la présence du où dans cette sous-classe des pronoms n'est pas systématique. Il n'apparaît que dans un peu moins de la moitié des publications proposant une liste de pronoms relatifs ${ }^{4}$. Nous ne décelons aucune chronologie particulière. Le rejet de la notion de lieu n'est pas explicite, seuls Jacquet (1848:18), Duchêne (1850 : 13) et Olivier (1857:30) précisent que le pronom relatif où a le sens de auquel, l'associant ainsi aux noms de choses et rejetant (certes, de façon peu catégorique) la notion sémantique de lieu.

où n'est pronom relatif que quand il a le sens de auquel (Duchêne, $1850: 13$ )

On constate que l'incidence notionnelle est présente, mais qu'elle n'est pas foncièrement entérinée par les grammaires de la période étudiée. L'opposition à une répartition notionnelle entre pronoms et adverbes ne rencontre cependant pas pour autant de vives critiques. En effet, les grammairiens qui s'opposent plus ou moins fermement à une distinction catégorielle reposant sur la signification sont rares (Jouannet, 1843 ; Jullien, 1854).

Cette proposition, de faire des mêmes mots à la fois deux espèces, selon les mots auxquels ils se rapportent, est contraire à toutes les bonnes habitudes en grammaire. $E n, y$, le, ne changent pas de nature parce qu'on les rapporte à des mots différents, pas plus que $i l$, elle; pas plus que tout adjectif, etc. [...]. D'un autre côté, c'est la première fois peut-être qu'on ferait de où un pronom, et sans doute un pronom de lieu; presque tous les grammairiens y ont vu un adverbe de lieu. (Jullien, $1854: 64-65$ )

Les grammairiens rangent ce mot [où] parmi les adverbes. Un raisonnement très simple va prouver que c'est une erreur, et que ce mot, avec l'accent grave, est toujours pronom. (Jouannet, $1843: 23$ )

\section{Les circonstances liées à l'adverbe}

Au-delà de l'opposition personne-chose versus lieu, qui fonde partiellement la distinction entre un pronom et un adverbe, il convient d'analyser les notions sémantiques qui accèdent au rang de circonstances au cours de la période couverte par le corpus. En effet, cela permettra de voir le statut de la signification dans la définition de l'adverbe, mais aussi les liens qui se tissent avec la fonction qui lui est intimement associée, celle de complément circonstanciel.

La signification occupe une place prépondérante dans la classe de l'adverbe puisque la grande majorité des ouvrages du corpus proposent un classement des adverbes par sens. Le seul classement concurrent, bien qu'il se situe nettement en retrait du précédent, est tout simplement celui par ordre alphabétique. Les autres classements sont rares, on relève essentiellement le classement morphologique : ainsi Gilard (1837) et Bentz (1838) distinguent-ils les adverbes simples des composés. Landais (1856) présente successivement le classement morphologique et sémantique. Bon nombre de grammairiens font également allusion à des adverbes qui sont morphologiquement des adjectifs.

Enfin, certains adjectifs sont quelquefois employés comme adverbes; on dit: chanter

juste, parler bas, voir clair, rester court, frapper fort, sentir bon, etc. (Jullien, 1852 :

La notion de circonstance n'apparaît pas explicitement liée au classement sémantique des listes d'adverbes (ce qui est différent de la définition même de l'adverbe). Deux auteurs font exception et lient les deux notions lors de la présentation du classement des adverbes. Il s'agit de Sardou (1841) et de Colart (1846), ce dernier établissant d'ailleurs un lien plus fort avec la notion de complément circonstanciel et les questions qui permettent d'identifier celui-ci. 
Les adverbes expriment une circonstance : $1^{\circ}$ De manière $[\ldots] .2^{\circ} \mathrm{D}$ 'ordre, de rang

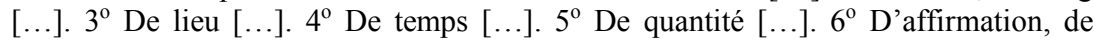

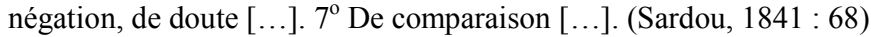

On subdivise les adverbes en huit classes, selon leur sens, les circonstances qu'ils expriment; les cinq premières [temps, lieu, manière, quantité, motif] donnent seules lieu à des questions. (Colart, $1846: 38$ )

Sur les 47 publications proposant un classement sémantique des adverbes, les sens systématiquement cités sont le lieu, le temps et la quantité. La manière est presque toujours présente. Elle semble avoir fait son chemin dans la description de l'adverbe, puisque, comme l'ont constaté Bouard et Fournier (2013), la manière était absente chez la plupart des grammairiens du XVIe et du XVIIe siècle et s'est peu à peu positionnée au sein des compléments circonstanciels.

Les autres notions n'apparaissent pas comme des incontournables. Si l'affirmation, la négation et l'ordre sont très bien représentés, la comparaison est déjà moins fréquente. On remarquera que la notion de motif, autrement dit la cause ou le but, n'apparaît qu'exceptionnellement dans le corpus, chez Colart (1846). On notera également que l'interrogation est en cours d'association avec la notion adverbiale. On la trouve déjà chez Villemeureux (1838) et Beudant (1840), mais elle est davantage présente à partir des années 1860. De plus, toutes les notions proposées n'ont pas le même statut: ainsi le lieu, le temps, la quantité, la manière ne caractérisent pas un verbe de la même manière que l'ordre, l'affirmation, la négation ou l'exclamation.

\begin{tabular}{|ll|c|}
\hline \multicolumn{2}{|c|}{ Notions circonstancielles } & Nombre de publications \\
\hline Lieu & (ici, là, dehors, etc. $)$ & $47 / 47$ \\
\hline Temps & (hier, jadis, toujours, etc. $)$ & $47 / 47$ \\
\hline Quantité $\quad$ (beaucoup, peu, trop, etc. $)$ & $47 / 47$ \\
\hline Manière & (poliment, à tort, etc. $)$ & $45 / 47$ \\
\hline Affirmation & (certes, vraiment, etc. $)$ & $38 / 47$ \\
\hline Négation & (ne point, nullement, etc. $)$ & $38 / 47$ \\
\hline Ordre & (premièrement, d'abord, etc. $)$ & $38 / 47$ \\
\hline Comparaison & (plus, mieux, autant, etc. $)$ & $30 / 47$ \\
\hline Doute & (sans doute, etc. $)$ & $14 / 47$ \\
\hline Interrogation & (combien, où, quand, etc. $)$ & $10 / 47^{5}$ \\
\hline Exclamation & (que, combien, etc. $)$ & $2 / 47$ \\
\hline Motif & (pour rire, de rage, etc. $)$ & $1 / 47$ \\
\hline
\end{tabular}

Tableau 1. Les sens qui permettent de classer les adverbes

\section{Les mots interrogatifs}

Le procédé des questions pour identifier les régimes direct et indirect est en place depuis Restaut (1732) (qui ? quoi ? à qui ? à quoi ? de qui ? de quoi ? par qui ? par quoi ?), mais, au cours du $\mathrm{XIX}^{\mathrm{e}}$ siècle, apparait une série d'autres emplois interrogatifs (avec qui ? avec quoi? dans quoi? vers quoi? où ? quand? comment? combien? pourquoi ?, etc.) qui vont peu à peu se répartir entre le complément indirect et le complément circonstanciel, dont la définition est en cours de modification (Piron 2012a, b). D'une part, la liste des mots interrogatifs comme procédés d'identification est en cours de formation; d'autre part, l'association stricte de ces mots interrogatifs soit avec le complément indirect, soit avec le complément circonstanciel est en cours de fixation. 
Puisque les mots interrogatifs sont en train d'acquérir un statut capital dans l'identification des compléments, il est intéressant d'étudier comment les grammaires du corpus présentent ces mots au sein des chapitres consacrés aux parties du discours.

\subsection{Les pronoms interrogatifs}

Le procédé des questions au moyen de qui et de quoi pour trouver le complément direct en particulier, mais aussi le complément indirect est bien implanté à l'époque couverte par le corpus, or les pronoms interrogatifs ne constituent pas couramment une sous-classe au sein des pronoms. Seize publications la proposent ${ }^{6}$ et autant de grammairiens considèrent plutôt que les pronoms relatifs (parfois appelés pronoms conjonctifs) servent à interroger ${ }^{7}$. Une certaine ambiguïté peut s'installer, les auteurs nommant alors les relatifs pronoms interrogatifs sans pour autant accorder un statut aux interrogatifs puisqu'ils ne figurent pas dans les sortes de pronoms généralement énumérées au début du chapitre consacré à cette partie du discours.

Un certain nombre de pronoms relatifs servent pour interroger : on les appelle alors pronoms interrogatifs. (Larive \& Fleury, $1883: 69$ )

La définition des pronoms servant à l'interrogation repose sur une notion sémantico-syntaxique, l'absence d'antécédent. Celle-ci est parfois associée à la définition notionnelle du pronom en tant que représentant une personne ou une chose (quelle personne? quelle chose ?), le distinguant ainsi de l'adverbe.

Le pronom interrogatif est celui qui n'a pas d'antécédent. (Rostagny, $1840: 5$ )

Les pronoms relatifs qui, que, quoi, lequel sont quelquefois interrogatifs [...] ils n'ont point d'antécédent et peuvent se tourner par quelle personne ou quelle chose? (Gavet, $1859: 25)$

Les pronoms interrogatifs n'ont pas d'antécédent, et peuvent toujours se tourner par quelle personne? quelle chose? (Sommer, $1861: 32$ )

Les pronoms interrogatifs pouvant être paraphrasés par quelle personne ? quelle chose ?, le statut de où comme pronom interrogatif est compromis. Seuls Landais (1856), Jouannet (1843), Colart (1846) et Descamps (1852) citent le où dans leur liste de pronoms interrogatifs.

\section{Je considère toujours où comme pronom [...] les pays où il pleut rarement, le village} d'où il s'enfuit; le but où l'on tend; où fuir ? Où me cacher? (Colart, 1846 : 14)

Cette proposition semble être limitée dans le temps. Il est étonnant de ne pas la trouver davantage chez les grammairiens qui considèrent que les pronoms relatifs (excepté dont) servent de pronoms d'interrogation. Or le classement du où comme pronom relatif, même s'il n'est pas systématique dans le corpus, semble moins contesté puisque 30 publications l'adoptent.

\subsection{Les adverbes interrogatifs}

Les adverbes interrogatifs apparaissent pour la première fois dans le corpus en 1838 chez Villemeureux, puis en 1840 chez Beudant. Il faut attendre les années 1850 pour que la notion soit reprise, mais elle est surtout présente dans les années 1860 et semble ensuite faiblir puisqu'on ne la retrouve que chez Brachet $(1874)^{8}$. Parmi les auteurs qui ne proposent pas une sous-classe d'adverbes interrogatifs, certains spécifient qu'une série d'adverbes servent à former une interrogation (Perron 1848, Poitevin 1856, Landais 1856, Gallien 1868).

On fait la question par un adverbe de manière (comment?), ou de lieu (où ? d'où ? par où ?), ou de temps (quand?), ou de cause (pourquoi ?). (Gallien, $1868: 28$ )

D'autres encore (Henry, 1860 ; Sommer, 1861 ; Chassang, 1881) en font une subdivision à l'intérieur des sous-classes sémantiques d'adverbes (lieu, temps, etc.). 


\begin{abstract}
Il y a un adverbe de lieu interrogatif, qui est : où ? [...] Il y a un adverbe de temps interrogatif, qui est : quand? [...] Il y a deux adverbes de manière interrogatifs, qui sont : pourquoi ? comment ? [...] Il y a un adverbe de quantité interrogatif, qui est : combien? [...] (Chassang, $1881: 167-172)$
\end{abstract}

Les termes où, quand, comment, combien et pourquoi revêtent une importance capitale dans le système fonctionnel puisqu'ils commencent, dans les années 1840-1860, à être associés au complément circonstanciel, et le sont même également au complément indirect (Piron, à paraître). Ces mots sont utilisés dans des questions qui acquièrent le statut de méthode d'identification de compléments du verbe. Après une période de flottement entre complément indirect et complément circonstanciel, le second sera associé aux questions où, quand, comment, combien et pourquoi. Dans notre corpus, l'importance de ces mots est en train de grandir puisqu'ils apparaissent dans la classe des adverbes (la classe liée par excellence au complément circonstanciel), mais leur statut adverbial et leur importance n'ont pas encore atteint leur apogée puisqu'on ne les trouve pas systématiquement dans les listes d'adverbes.

Parmi ces cinq mots, celui qui est, de loin, le plus souvent cité parmi les adverbes est le où. À l'inverse, le moins cité comme adverbe est le pourquoi. Cela n'est pas étonnant quand on sait que l'interrogatif quoi a longtemps été, et est encore facilement associé dans les années 1840, à une vaste liste de prépositions pour l'identification du complément indirect (Piron, 2012b). On trouve ainsi encore dans les années 1840 des questions en pour quoi (en deux mots), aux côtés de à quoi, de quoi, par quoi, etc. (par exemple, Belèze, 1842 ; Jouannet, 1843).

Les rapports sont inversés lorsqu'il s'agit de considérer ces cinq mots comme des adverbes interrogatifs : comment, quand et pourquoi sont plus aisément considérés comme adverbes interrogatifs que où et combien. Les cinq mots ne forment pas encore un ensemble très soudé puisque seuls quatre auteurs du corpus proposent la liste qui deviendra la liste complète des interrogatifs adverbiaux où, quand, comment, combien et pourquoi (Villemeureux, 1838 ; Michel \& Rapet, 1860 ; DMD, 1866 ; Chassang, 1881).

\begin{tabular}{|c|c|c|}
\hline \multirow{2}{*}{$\begin{array}{l}\text { Mots en cours d'association } \\
\text { avec le complément } \\
\text { circonstanciel }\end{array}$} & \multicolumn{2}{|c|}{ Nombre de publications } \\
\hline & $\begin{array}{c}\text { Mots figurant dans la liste } \\
\text { des adverbes }\end{array}$ & $\begin{array}{l}\text { Mots figurant dans la liste } \\
\text { des adverbes interrogatifs }\end{array}$ \\
\hline où? & $68 \%(53 / 78)$ & $13 \% \quad\left(7^{9} / 53\right)$ \\
\hline combien? & $55 \%(43 / 78)$ & $26 \% \quad\left(11^{10} / 43\right)$ \\
\hline comment? & $44 \%(34 / 78)$ & $38 \% \quad\left(13^{11} / 34\right)$ \\
\hline quand? & $35 \%(27 / 78)$ & $44 \% \quad\left(12^{12} / 27\right)$ \\
\hline pourquoi? & $23 \%(18 / 78)$ & $67 \% \quad\left(12^{13} / 18\right)$ \\
\hline
\end{tabular}

Tableau 2. Les adverbes en cours d'association avec le complément circonstanciel

L'association entre la nature adverbiale et les significations liées à ces mots interrogatifs se fait plus forte et transparaît chez Henry (1860), qui ne propose pourtant pas d'adverbes interrogatifs. On remarquera l'absence de pourquoi.

On reconnaît, en général, qu'un mot invariable est un adverbe appartenant à l'une des quatre premières espèces (temps, lieu, manière, quantité), qui sont de beaucoup les plus nombreuses, s'il répond à l'une des questions : Quand? Où ? Comment? Combien? (Henry, 1860 : 125)

Un tel procédé de reconnaissance des adverbes est cependant loin d'être l'apanage de la grammaire du $\mathrm{XIX}^{\mathrm{e}}$ siècle. Ainsi, Restaut (1732) classait-il ses adverbes, en omettant les adverbes de motif et l'interrogation en pourquoi.

1. Les adverbes de tems qui répondent à la question quand ? [...] 2. Les adverbes de lieu ou de situation, qui répondent à la question où ? [...] 4. Les adverbes de 
quantité ou de nombre, qui répondent à la question combien ? [...] 7. Les adverbes de qualité ou de maniere, qui répondent à la question comment? (Restaut, 1732:87-88)

\section{Conclusion}

L'étude présentée s'est attachée aux classements des pronoms et des adverbes, et plus particulièrement à une liste de mots que la grammaire du $\mathrm{XIX}^{\mathrm{e}}$ siècle associe aux procédés de questions mis au point pour distinguer les compléments directs, indirects et circonstanciels (qui, que, quoi, où, quand, comment, combien, pourquoi). Il a ainsi été constaté qu'au-delà des définitions reposant sur les notions syntaxicosémantiques de modification et de remplacement, les notions sémantiques de personne et de chose étaient attachées à la classe du pronom tandis que celles de circonstance (notamment le lieu) l'étaient à l'adverbe. Pour preuve, il été constaté une certaine ambivalence de classement entre adverbe et pronom des mots en, $y$ et où selon qu'ils signifiaient un lieu ou une chose, une personne.

L'opposition personne, chose versus circonstances est également importante dans le cas des interrogatifs. En effet, les pronoms interrogatifs qui, que, quoi sont dits substituables par les expressions quelle personne? quelle chose? Le critère notionnel est aussi utilisé pour les adverbes, mais il est éclaté en une série de notions. Les adverbes interrogatifs où, quand, comment, combien, pourquoi semblent cependant difficilement se frayer un chemin au sein de la liste des adverbes. Même les notions systématiquement associées à l'adverbe (lieu, temps, quantité) ou presque systématiquement (manière) ne présentent pas automatiquement un adverbe interrogatif : par exemple, où n'apparaît dans la liste des adverbes que dans $68 \%$ des cas et n'est considéré comme adverbe interrogatif que dans $13 \%$ des cas.

Malgré ces constats, la recherche permet d'affirmer que les mots interrogatifs font partie de l'architecture grammaticale de la période étudiée, même s'ils ne sont pas encore systématiquement présentés ni regroupés. Les circonstances qui permettent de classer les listes des adverbes proposées par les grammairiens ne sont pas strictement établies au cours de la période 1835-1883. Le lieu, le temps, la quantité et la manière font partie des canons circonstanciels, mais les autres notions présentent des fluctuations. Quant à la notion de cause (trouvée uniquement chez Colart (1846) sous l'appellation motif), elle ne fait pas encore partie du classement sémantique des adverbes, ni celle de but (d'ailleurs encore associée au complément indirect). Ainsi, en 1860, le pourquoi ? ne fait pas encore systématiquement partie des questions pour reconnaître le complément circonstanciel et semble encore partiellement attaché au complément indirect. Il est donc cohérent que les grammaires consignent plus rarement pourquoi comme adverbe que où, combien, et comment.

Il est probable que la place des mots interrogatifs où, quand, comment, combien et pourquoi se renforce au fil des décennies suivantes en tant qu'adverbes et plus spécifiquement en tant qu'adverbes interrogatifs lorsque les questions associées au complément circonstanciel se seront fixées et seront devenues intimement liées à la définition de cette fonction.

\section{Références bibliographiques}

\section{Références secondaires}

Benveniste, É. (1966) Problèmes de linguistique générale. Paris : Gallimard.

Bouard, B. \& Fournier, J.-M. (2013). Complément, adverbe et expression de la manière dans les grammaires françaises, XVIe-XIXe siècles. In Scolia 27 : 31-52.

Chervel, A. (1977). Et il fallut apprendre à écrire à tous les petits Français. Histoire de la grammaire scolaire, Paris : Payot.

Chervel, A. (1979). Rhétorique et grammaire, petite histoire du circonstanciel. Langue française, $n^{\circ} 41$, numéro spécial sur la grammaire traditionnelle, 5-19. 
Choi-Jonin, I. (2001). Pronom ou adverbe : où situer les interrogatifs spatio-temporels ? in Colombat, B., Savelli, M. Métalangage et terminologie linguistique. Actes du colloque international de Grenoble (Université Stendhal Grenoble III, 14-16 mai 1998). Leuven - Paris - Sterling, Virgina : Peeters, pp. 977-991.

Creissels, D. (1995). Éléments de syntaxe générale. Paris : PUF.

Gross, G. (2012). Manuel d'analyse linguistique. Paris : Septentrion.

Gross, M. (1986). Grammaire transformationelle du français. Vol. 3, Syntaxe de l'adverbe. Paris : ASSTRIL.

Guimier, C. (1996). Les adverbes du français : le cas des adverbes en -ment. Paris : Ophrys.

Piron, S. (2012a). Les compléments prépositionnels dans les grammaires françaises de la première moitié du XIXe siècle. In Neveu, F., Muni Toke, V., Blumenthal, P., Klinger, T., Ligas, P., Prévost, S., Teston-Bonnard, S. (dir.) CMLF 2012 - Troisième Congrès mondial de linguistique française. Lyon, 4-7 juillet 2012. Institut de linguistique française : EDP Sciences, pp. 773-788.

Piron S. (2012b) The treatment of prepositional phrases in French grammars during the first half of the 19th century. Henry Sweet Society for the History of Linguistic Ideas. Annual colloquium, 30 août - 1er septembre 2012. Aarhus University, Danemark.

Piron S. (2012c) "Évolution des classements verbaux : verbes neutres, intransitifs et transitifs indirects » in Colomabt B., Fournier J.-M. et Raby V. (dir.) Vers une histoire générale de la grammaire française ? Matériaux et perspectives. Paris, Honoré Champion, pp. 759-779.

Piron, S. (à paraître). Les syntagmes prépositionnels au tournant de la deuxième grammaire scolaire française. In Fryba, A.-M., Antonelli, R., Colombat, B. (dir.) Actes du XXVIIe Congrès international de linguistique et de philologie romanes (Nancy, 15-20 juillet 2013). Section 15: Histoire de la linguistique et de la philologie. Nancy, ATILF.

Ruwet, N. (1968 [1967]). Introduction à la grammaire générative. Paris : Plon.

\section{Références primaires}

Alix (1844). Programme ou tableaux de grammaire française. Angers : Cosnier et Lachèse.

Alix et Davau, R. (1849 [1843]). Grammaire française rédigée d'après les principes de l'Académie, Paris : Hachette ; Angers : Cosnier et Lachèse.

Anonyme (1861). Préparation à la grammaire française ou la prononciation, L'orthographe d'usage et les dix parties $d u$ discours. Metz: Warion.

Anonyme (1865). Grammaire française d'après les meilleurs auteurs. L'école mutuelle, cours complet d'éducation populaire. Paris : Bureaux de la publication.

Anonyme, 1858 ( $2^{\mathrm{e}}$ éd.). Grammaire française des maîtres et des élèves spécialement à l'usage des écoles normales primaires et des classes élémentaires des colléges; précédée d'une introduction relative aux règles de la prononciation. Paris : L. F. Hivert.

Beauzée, N. (1767). Grammaire générale ou exposition raisonnée des éléments nécessaires du langage, pour servir de fondement à l'étude de toutes les langues. 2 tomes. Paris : J. Barbou.

Belèze, G. (1843). Abrégé de la grammaire française pour le premier âge avec exercices élémentaires. Paris : Jules Delalain.

Belèze, G. (1862). Grammaire française selon les principes de l'Académie avec questionnaires. Paris: Jules Delalain.

Bentz, L. (1838). Éléments abrégés de la grammaire française, à l'usage des écoles. Paris : Librairie ecclésiastique, classique et élémentaire de H. Delloye.

Ber, F.-J. (1854). Méthode d'analyse grammaticale réduite à sa plus simple expression. Metz.

Bertheau, A. (1845). Grammaire française élémentaire. Montrichard : chez l'auteur. 
Bescherelle, H. (1856, 24éd.). Grammaire française élémentaire et pratique à l'usage des maisons d'éducation, des écoles primaires d'enfants et d'adultes des deux sexes, ouvrage rédigé sur un plan entièrement neuf. Paris : Ch. Fouraut.

Beudant, S. F. (1840). Nouveaux éléments de grammaire française. Paris : Pitois-Levrault et Cie.

Boniface, A. (1848 [1836]). Abrégé de la grammaire française méthodique et raisonnée, adoptée par le Conseil de l'Université pour les classes élémentaires des colléges et les écoles primaires et normales. Paris : Delalain.

Bonneau et Lucan (1839). La grammaire selon l'Académie, revue par M. Michaud. Paris : chez les auteurs et chez Gosselin.

Brachet, A. (1876, $4^{\mathrm{e}}$ éd. [1874]). Nouvelle grammaire française fondée sur l'histoire de la langue à l'usage des établissements d'instruction secondaire. Paris, Hachette.

Brun, M.-L., Bertrand C. (1854). Principes de grammaire française. Béziers : Bertrand.

Canihac-Bélair, P. (1861). Règles de l'orthographe française ou grammaire. Paris: Paul Dupont ; Bordeaux: Muller; Toulouse : Bourdin.

Capelle-Grimbert (1842). Grammaire française raisonnée, ou petite grammaire des grammaires. Lille: De Vanackere.

Caplain, H et Dupont, P.-A.-B. (1838 [1831]). Grammaire française classique. Saint-Quentin : Ribaud-Leradde et Doloy.

Chapsal, M. (1841). Syntaxe française ou étude raisonnée de toutes les difficultés que présente notre langue sous le rapport syntaxique. Paris : Maire-Nyon, Roret, Hachette, Delalain.

Chassang, A. (1881, 6 éd. [1880]). Nouvelle grammaire française. Cours supérieur avec des notions sur l'histoire de la langue et en particulier sur les variations de la syntaxe du XVIe au XIXe siècle. Paris : Garnier Frères.

Clouzet, P. - A. (1856 [1835]). Grammaire française sur un plan entièrement nouveau, divisée en cinq parties: conjugaison, analyse grammaticale, analyse logique, orthographe de principes, et orthologie. Paris : Fonteney et Peltier; Bordeaux : chez l'Auteur.

Colart, L. S. (1846). Nouvelle grammaire française logique et intuitive, en tableaux synoptiques avec textes explicatifs. Paris : chez l'auteur.

D. M. D. (1866, 4 éd. [1851]). Manuel de grammaire français à l'usage des écoles primaires de la Société de Marie. Lons-le-Saunier.

D'Assier, A. (1864). Grammaire abrégée de la langue française d'après la grammaire générale des langues indoeuropéennes pour faciliter l'étude des langues classiques. Ouvrage destiné aux élèves des écoles primaires, des Colléges et des écoles normales. Paris, Durand.

Delavenne, Père H. (1868). Grammaire de la langue française d'après l'Académie et les meilleurs écrivains. Grammaire complète. Amiens : Alfred Caron fils ; Paris : Poussielgue-Rusand.

Descamps, J. (1852). Abrégé de la grammaire française. Douai : Adam D’Aubers.

Dessiaux, J. (1838 [1837]). Grammaire française, analytique et pratique à l'usage des écoles élémentaires. Paris : Beaujouan.

Dorion, E. (1855). La grammaire française ramenée à des principes rigoureux; théorie nouvelle. Amiens : LenoelHerouart.

Duchêne, L. (1850). Abrégé de la grammaire française des écoles primaires élémentaires, sur un plan tout-à-fait nouveau et très-méthodique, avec des exercices analytiques, orthographiques et syntaxiques, gradués sur chaque règle et suivis de récapitulations, ouvrage mis à la portée des plus jeunes élèves. Boulogne-sur-mer: chez l'auteur.

Dunglas, C. (1855). Abrégé de grammaire française. Le Puy : Alexis Guilhaume.

F. M. S. B. (1864). Grammaire française élémentaire à l'usage des écoles dirigées par les frères de l'instruction chrétienne de Saint-Gabriel. Poitiers : Henri Oudin.

F. P. B. \& F. I. C. (1877). Grammaire française. Cours moyen et supérieur. Tours : Alfred Mame et fils ; Paris : Poussielgue frères. 
Fricadel-Dubiez, A. (1849). Cours élémentaire de grammaire française, rédigé d'après une méthode nouvelle qui permet l'emploi de la forme dialogique et suivi d'exercices orthographiques et de modèles d'analyse grammaticale. Paris : librairie enfantine et juvénile de Pierre Maumus.

Gallien, J. V. (1868). Grammaire française analytique et pratique. Paris : Larousse et Boyer.

Ganeval, L. (1862). Grammaire raisonnée de la langue française. Paris : Durand, Fouraut.

Gavet, N. (1859, $3^{\mathrm{e}}$ éd.) Grammaire française. Paris : Paul Dupont.

Gilard, M. (1837). Grammaire pratique, analytique et théorique de la langue française, divisée en trois parties. Première partie : prononciation grammaticale et prosodique. Deuxième partie : classification et nature des mots démontrée par l'analyse grammaticale subdivisée d'après le plan méthodique de l'ouvrage; théorie nouvelle des verbes. Troisième partie : participes; accord, significations, orthographe et emploi des mots; nouveau moyen d'apprendre par l'analyse logique la construction des mots, des propositions et des phrases; inversions; ellipses; pléonasmes; gallicismes; précis de versification. Paris : Daubrée.

Girard, F. (1862). Rudiments de la langue française. Grammaire des enfants, à l'usage des écoles primaires des deux sexes. Saint-Maixent : Reversé.

Gresse, A. (1852). Grammaire française méthodique, à l'usage des écoles primaires et des classes élémentaires des collèges. Paris : Hachette, Delalain, Langlois et Leclerc, Ducrocq; Valence : chez l'auteur.

Guérard, M. (1853 [1851]). Cours complet de langue française (théorie et exercices), première partie : grammaire élémentaire d'après Lhomond, livre de l'élève. Paris : Dezobry et Magdeleine.

Guerrier de Haupt, A. (1842). Abrégé de la grammaire française, d'après les principes de l'Académie. Paris : Hachette-Tetu ; Amiens : Prévost-Allo.

Henry, A. (1860). Précis de logique grammaticale spécialement appliqué à la langue française, ouvrage dédié aux jeunes maîtres. Nancy : Grimblot et Cie ; Paris : Hachette et Cie.

Huré, H. (1865, $2^{\mathrm{e}}$ éd. [1863]). Grammaire française. Premier degré. Éléments. Paris : Fred. Cantel.

Jacquet, F. (1848). Grammaire française élémentaire, suivie d'un traité d'analyse grammaticale et d'exercices orthographiques, à l'usage des écoles primaires. Lyon : veuve Ayné.

Jonette, H. (1869). Grammaire des commençants ou éléments de la grammaire française à l'usage des très-jeunes enfants. Paris : Eugène Belin.

Jouannet, P.-C. (1843). Traité d'analyse grammaticale, précédé de plusieurs observations sur les dix parties du discours et d'une discussion sur l'analyse. Paris : chez les principaux libraires; Bordeaux: Ch. Lawalle et Chaumas-Gayet.

Jourdan, R., Castegnier, H. (1863). Grammaire française. Paris : Étienne Giraud ; Nîmes : Louis Giraud.

Jullien, B. (1852). Traité complet de grammaire française, comprenant avec les règles fondamentales et particulières de notre langue l'étude des gallicismes les plus usités. Paris : Hachette.

Jullien, B. (1854). Explication des principales difficultés de l'enseignement de la grammaire, à l'usage de toutes les personnes chargées d'instruire ou d'interroger les enfants. Paris: Hachette.

Laglaine, P. (1864). Grammaire française et dictées. Paris : Victor Sarlit.

Lambert, A.-F. (1846). Grammaire rudimentaire de la langue française. Paris : chez l'auteur.

Lamotte, L., Bescherelle, L. N. (1841). Grammaire de l'Académie, ou Principes de grammaire française, fidèlement extraits de la dernière édition du Dictionnaire de l'Académie. Paris : Delloye.

Landais, N. (1856 [1835]). Grammaire Générale des Grammaires Françaises présentant la solution analytique, raisonnée et logique de toutes les questions grammaticales anciennes et modernes. Paris : Didier.

Larive et Fleury (1883 [1875]). La troisième année de grammaire, révision et compléments de grammaire, formation des mots - style et composition - littérature, histoire littéraire (biographies et morceaux choisis), avec exercices et lexique, ouvrage rédigé conformément aux principes de la grammaire historique, à l'usage des écoles commerciales, des élèves de l'enseignement secondaire spécial et des pensionnats de demoiselles. Partie du maître. Paris : Armand Colin et Cie. 
Larousse, P. (1852 [1850]). La lexicologie des écoles. Cours complet de langue française et de style divisé en trois années et rédigé sur un plan entièrement neuf. $1^{\text {re }}$ année, grammaire élémentaire et lexicologie, partie de l'élève. Paris : Larousse et Boyer.

Laurent, A. (1864). L'ABC de la grammaire ou introduction à l'étude de la grammaire française. Paris: E. Ducrocq.

Leclair L. (1859). Grammaire de la langue française ramenée aux principes les plus simples. Grammaire complète. Paris : Eugène Belin.

Michel, L.-C., Rapet, J.-J. (1860). Principes de grammaire française. Paris : Dezobry, Magdeleine et Cie.

Ministère de la Guerre (1866, $2^{\mathrm{e}}$ éd.). Cours de grammaire française pour les $1^{r e}, 2^{e}$ et $3^{e}$ sections. Paris : librairie militaire, J. Dumaine.

Negrin, É. (1864). Grammaire française des gens du monde. Nice : Imprimerie administrative.

Olivier, Th. (1857). Grammaire française élémentaire, comprenant des notions développées sur l'orthographe, l'analyse grammaticale et l'analyse logique, à l'usage des écoles primaires et moyennes. Paris, Tournai: Casterman.

Père de la Compagnie de Jésus, un (1856). Grammaire française élémentaire rédigée sur un nouveau plan par un père de la Compagnie de Jésus. Toulouse : Édouard Privat ; Paris : Pélagaud et Cie ; Lyon : Pélagaud et Cie.

Perron, F. (1848). Nouveaux principes de grammaire française, rédigés d'après les éléments de grammaire générale du même auteur, à l'usage des colléges, des institutions et des écoles primaires. Paris: Didot, Chamerot, Lecoffre ; Besançon : Outhenin Chalandre fils.

Perrot D'Ablancourt, M. (1838). Nouvel abrégé de grammaire française, suivi d'un traité d'orthographe pratique, à l'aide duquel on peut apprendre à écrire convenablement et seul la plupart des mots de notre langue. Poitiers : Fradet.

Poitevin, P. (1845 [1843]). Étude raisonnée de la syntaxe française, ouvrage rédigé sur un plan entièrement neuf où sont exposées et résolues toutes les difficultés que présente notre langue sous le rapport syntaxique. Paris: Firmin Didot Frères, Désobry et Magdeleine, Maire-Nyon, Jacques Lecoffre.

Poitevin, P. (1856). Grammaire générale et historique de la langue française présentant l'étude et l'analyse de la formation, des développements et des variations de notre idiome national depuis son origine jusqu'à nos jours, Tomes 1 et 2. Paris : Bureaux du magasin pittoresque.

Poitevin, P. (1869 [1844]). Cours théorique et pratique de langue française, ouvrage rédigé sur un plan entièrement neuf. Grammaire complète, théorie et application. Nouvelle édition. Paris : Firmin-Didot frères, Jacques Lecoffre et Cie, Delagrave et Cie.

Restaut, P. (1732). Abrégé des principes de la grammaire françoise. Paris : Jean Desaint.

Roche, A. (1860 [1852)]. Grammaire française. Adoptée par le Conseil Impérial de l'Instruction publique pour les Lycées et les Collèges de France. Nouvelle édition (à l'usage des Anglais). London, Edinburgh : Williams and Norgate.

Rostagny, M. (1840). Grammaire et orthographe simplifiées, mises à la portée de toutes le sintelligences. Arles: Garcin.

Sardou, A. L. (1840 [1837]). Leçons de grammaire française et exercices de style. Paris, Hachette.

Sardou, A. L. (1841). Cours de grammaire française. Paris, Hachette.

Sommer, É. (1861). Cours complet de grammaire française à l'usage des établissements d'instruction secondaireet des écoles primaires supérieures. Paris : Hachette.

Temporal, J. L. (1844). Atlas grammatical ou tableaux synoptiques de la grammaire française. Châlon-sur-Saône : chez tous les libraires, chez Brille et Guichard et chez l'auteur.

Van Hollebeke, B. (1883, $27^{\mathrm{e}}$ éd. [1865]). Éléments de la grammaire française à l'usage des écoles primaires. Lexicologie. Namur : Wesmael-Charlier.

Vigneau, S. M., (1846). Éléments de grammaire française simplifiée, suivis d'exercices analytiques, orthographiques, historiques et géographiques, propres à développer l'intelligence et la mémoire des élèves. Paris : Hachette ; Chartres : Garnier ; Pont-Audemer : Dugas-Lecomte, chez l'auteur. 
Villemeureux, C. (1838). Grammaire française de Lhomond, développée et complétée par Constant Villemeureux. Paris : librairie classique de Madame veuve Maire-Nyon.

Willequet, R. (1842). Nouvelle grammaire française. Gand : Lebrun-Devigne.

${ }^{1}$ Clouzet (1835), Boniface (1836), Caplain et Dupont (1838), Dessiaux (1838), Bonneau et Lucan (1839), Chapsal (1841), Lamotte et Bescherelle (1841), Willequet (1842), Davau et Alix (1849), Poitevin (1869), Jouannet (1843), Temporal (1844), Bertheau (1845), Lambert (1846), Jacquet (1848), Duchêne (1850), Roche (1860), Gresse (1842), Larousse (1852), Brun \& Bertrand (1854), Dorion (1855), Poitevin (1856), Père (1856), Bescherelle (1856), Anonyme-Hivert (1858), Anonyme (1858), Leclair (1859), Henry (1860), Anonyme-Metz (1861), Canihac-Bélair (1861), Jourdan et Castegnier (1863), FSMB (1864), D’Assier (1864), Négrin (1864), Delavenne (1868).

${ }^{2}$ Villemeureux (1838), Sardou (1840; 1841), Capelle-Grimbert (1842), Belèze (1843; 1862), Vigneau (1846), Colart (1846), Fricadel-Dubiez (1849), Guérard (1853), Ber (1854), Landais (1856), Olivier (1857), Sommer (1861), Girard (1862), Anonyme (1865), Huré (1865), F.P.B \& F.I.C. (1877).

${ }^{3}$ Les auteurs sont au nombre de 34 : Clouzet (1835), Sardou (1837), Gilard (1837), D'Ablancourt (1838), Dessiaux (1838), Bentz (1838), Willequet (1842), Bertheau (1845), Lambert (1846), Colart (1846), Jacquet (1848), Perron (1848), Duchêne (1850), Guérard (1851), Descamps (1852), Larousse (1852), Dunglas (1855), Olivier (1857), Anonyme chez Hivert (1858), Leclair (1859), Gavet (1859), Henry (1860), Canihac-Bélair (1861), Sommer (1861), Belèze (1862), Jourdan \& Castegnier (1863), Négrin (1864), Anonyme École mutuelle (1865), DMD (1866), Delavenne (1868), Brachet (1876), F.P.B. \& F.I.C. (1877), Chassang (1881), Larive \& Fleury (1883).

${ }^{4}$ Clouzet (1835), Desssiaux (1838), Lamotte \& Bescherelle (1841), Capelle-Grimbert (1842), Willequet (1842), Jouannet (1843), Belèze (1843), Temporal (1844), Vigneau (1846), Colart (1846), Lambert (1846), Jacquet (1848), Duchêne (1850), Descamps (1852), Larousse (1852), Landais (1856), Poitevin (1856), Bescherelle (1856), Olivier (1857), Roche (1860), Sommer (1861), Anonyme à Metz (1861), Belèze (1862), Négrin (1864), Gallien (1868), Poitevin (1869), F.P.B. \& F.I.C. (1877), Chassang (1881).

${ }^{5}$ Villemeureux (1838), Beudant (1840), Dunglas (1855), Michel \& Rapet (1860), Roche (1860 [1852]), Sommer (1861), Jourdan \& Castenier (1863), D’Assier (1864), D.M.D. (1866 [1851]), Brachet (1876 [1874]).

${ }^{6}$ Clouzet (1835), D’Ablancourt (1838), Villemeureux (1838), Rostagny (1840), Willequet (1842), Colart (1846), Dorion (1855), Anonyme chez Hivert (1858), Michel \& Rapet (1860), Roche (1860), Anonyme à Metz (1861), Sommer (1861), Anonyme École mutuelle (1865), Huré (1865), Chassang (1881).

${ }^{7}$ Père (1856), Bentz (1838), Gavet (1859), Sardou (1840), Olivier (1857), Alix (1844), Bescherelle (1844), Poitevin (1845, 1856), FSMB (1864), Van Hollebeke (1865), Ministère de la Guerre (1866), Jonette (1869), Brachet (1876), Chassang (1881), LArive \& Fleury (1883).

${ }^{8}$ Beudant (1840), Dunglas (1855), Roche (1860), Michel \& Rapet (1860), Sommer (1861), Jourdan \& Castegnier (1863), D’Assier (1864), DMD (1866), Brachet (1876).

${ }^{9}$ Villemeureux (1838), Beudant (1840), Michel \& Rapet (1860), DMD (1866), Sommer (1861), Chassang 1881.

${ }^{10}$ Villemeureux (1838), Dunglas (1855), Landais (1856), Michel \& Rapet (1860), Roche (1860), Jourdan \& Castegnier (1863), D’Assier (1864), Brachet (1876).

${ }^{11}$ Beudant (1840), Villemeureux (1838), Perron (1848), Landais (1856), Roche (1860), Michel \& Rapet (1860), Sommer (1861), Jourdan \& Castegnier (1863), D’Assier (1864), DMD (1866), Brachet (1876), Chassang (1881).

${ }^{12}$ Villemeureux (1838), Beudant (1840), Dunglas (1855), Landais (1856), Roche (1860), Michel \& Rapet (1860), Sommer (1861), D’Assier (1864), DMD (1866), Brachet (1876), Chassang (1881).

${ }^{13}$ Villemeureux (1838), Beudant (1840), Lamotte \& Bescherelle (1841), Perron (1848), Dunglas (1855), Roche (1860), Michel \& Rapet (1860), D’Assier (1864), DMD (1866), Brachet (1876), Chassang (1881). 